\title{
Seroprevalence and factors associated with seropositivity to Rift Valley fever virus in livestock
}

\section{Yusuf B. Ngoshe ${ }^{1}$, Lida Avenant ${ }^{1}$, MK Rostal' ${ }^{2}$, Karesh B. William², Janus T. Paweska ${ }^{3}$, Jansen van Vuren ${ }^{3}$, Claudia Cordel ${ }^{4}$, Veerle Msimang ${ }^{3,1}$, Peter N. Thompson ${ }^{1}$}

\author{
${ }^{1}$ Epidemiology Section, Production Animal StudiesStudy, University of Pretoria, Pretoria, Gauteng, South Africa, ${ }^{2}$ EcoHealth Alliance, NYC, New \\ York, United States, ${ }^{3}$ National Institute for Communicable Diseases, Pretoria, Gauteng, South Africa, ${ }^{4}$ Execuvet, Bloemfontein, Free State, \\ South Africa
}

\section{Objective}

The objective of this study was to estimate the prevalence of antibodies to RVFV in domestic cattle, sheep, and goats in a study area in the central interior of South Africa, and to identify factors associated with seropositivity.

\section{Introduction}

Rift Valley fever (RVF) is a mosquito-borne viral zoonosis. This study aimed to estimate the prevalence of antibodies to RVF virus (RVFV) in cattle, sheep, and goats in South Africa, near the 2010-2011 outbreak epicenter and identify factors associated with seropositivity.

\section{Methods}

A cross-sectional study was conducted during 2015-2016 within a $\sim 40,000 \mathrm{~km}^{2}$ region between Bloemfontein and Kimberley. Farms were selected using random geographic points with probability proportional to the density of livestock-owning households. Livestock were randomly sampled from the farm closest to each selected point. A questionnaire was used to collect information concerning animal, management, and environmental factors. Sera samples were screened for RVFV antibodies using IgG inhibition ELISA. Data were analyzed using multilevel logistic regression models.

\section{Results}

On 234 farms, 3,049 animals (977 cattle, 1,549 sheep and 523 goats) were sampled. Estimated RVF seroprevalence, adjusted for clustering and sampling weights, was $42.9 \%$ (95\% CI: 35.7-50.4\%) in cattle, $28.0 \%$ (95\% CI: $21.3-35.4 \%$ ) in sheep and 9.3\% (95\% CI: 5.8-13.9\%) in goats. Compared to animals $<2 \mathrm{y}$ of age, seroprevalence was higher in animals $2-4 \mathrm{y}(\mathrm{OR}=2.8$, $P<0.001)$ and $>4$ y old $(\mathrm{OR}=17.0, P<0.001)$. Seroprevalence was also higher on private vs. communal land $(\mathrm{OR}=4.3, P=0.001)$ and was positively associated with the presence of perennial rivers $(\mathrm{OR}=1.6, P=0.03)$ and seasonal pans $(\mathrm{OR}=1.8, P=0.005)$ on the farm. The odds of seropositivity was higher in domestic ruminants recently vaccinated between 2014-2015 (OR=2.1, $P=0.007)$ compared to those never vaccinated.

\section{Conclusions}

The presence of IgG antibody against RVFV among domestic ruminants, born after the most recent outbreak ( $<4 y$ category), and association with known RVF risk factors, indicates the possibility that viral circulation has occurred during the interepidemic period.

\section{Acknowledgement}

The authors acknowledge and are extremely grateful to all the farmers that participated in this study and to the state veterinarians and animal health technicians of Free state and Northern Cape Provinces for facilitating identification of farms and contacts with the farmers. We also thank Karissa Whiting for the development of the electronic mobile application for the survey data collection. The field and laboratory work for this project was funded by the Understanding Rift Valley Fever in South Africa Project, sponsored by the U.S. Department of Defense, Defense Threat Reduction Agency. The content of the information does not necessarily reflect the position or the policy of the federal government, and no official endorsement should be inferred. The EcoHealth Alliance, University of Pretoria and National Research Foundation are acknowledged for financial support. 